\title{
SURVEY ACCURACY AND SPATIAL RESOLUTION BENCHMARK OF A CAMERA SYSTEM MOUNTED ON A FAST FLYING DRONE
}

\author{
H. Meißner ${ }^{\mathrm{a}, *}$, K. Stebner ${ }^{\mathrm{a}}$, T. Kraft ${ }^{\mathrm{a}}$, M. Geßner ${ }^{\mathrm{a}}$, R. Berger ${ }^{\mathrm{a}}$ \\ ${ }^{a}$ Institute of Optical Sensor Systems, German Aerospace Center, 12489 Berlin, Germany
}

Commission TCI-Sensor Systems

KEY WORDS: Drones, Fixed-wing, Metric Camera System, Aerial Photogrammetry, Survey, Spatial Resolution

\begin{abstract}
:
Many drones are used to obtain high resolution imagery. Subsequent 3D object point derivation from images of these systems is an established technique. While rotor-craft drones are often used to capture fine, detailed structures and objects in small-scale areas fixedwing versions are commonly used to cover larger areas even far beyond line of sight. Usually, these drones fly at much higher velocities during data acquisition and therefore the according sensor requirements are much higher.

This paper presents the evaluation of a prototype camera system for fast flying fixed-wing drones. Focus of investigation is to find out if higher operating velocities, up to $100 \mathrm{~km} / \mathrm{h}$ during image acquisition, has any influence on photogrammetric survey and image quality itself. It will be shown that images, obtained by the presented camera system and carrier, do not suffer from motion blur and that the overall survey accuracy is approximately $1 / 4$ of ground sample distance.

Survey accuracy analysis is carried out using standard photgrammetric procedures using signaled control- and checkpoints and verifying their conformity in image space and object space.

Fundamentals of image quality will be introduced, as well asan approach to determine and evaluate motion smear of remote sensing senors (in theory and practical use case). Furthermore, it will be shown that the designed camera system mounted on a fixed-wing carrier does not suffer from motion smear.
\end{abstract}

\section{INTRODUCTION}

The rapid development of commercial drones has led to an increased availability of civilian solutions being reliable, safe and easy to operate. There are rotor-craft and fixed-wing designs. Most common are small rotor-craft drones (e.g. DJI, Yuneec, Intel) equipped with optical payloads. These systems are suitable for small-scale aerial imaging applications, e.g. optical measurement and documentation of buildings and industrial plants.

In comparison to rotor-craft drones, fixed-wing versions (e.g. Delair, senseFly, Quantum Systems) allow for longer flight times because of their gliding characteristics which makes it an ideal tool for large-scale mapping applications (Hein et al., 2019) and for LIDAR- and multi-spectral applications (Khan et al., 2017, Bakuła et al., 2018, Hruska et al., 2012)

Latest developments of rotor-craft and fixed-wing drones increasingly address the survey market. These drones are equipped with advanced GNSS receivers to improve position accuracy by enabling real-time kinematic (RTK) and post-processed kinematic (PPK) techniques leading to a more precise approximation for exterior orientation of aerial images. Survey accuracy of derived models from all these systems can be obtained using a geodeticphotogrammetric test field (Przybilla et al., 2019).

Regarding fixed-wing drones operating at typical velocities between $80 \mathrm{~km} / \mathrm{h}$ and $100 \mathrm{~km} / \mathrm{h}$ (rotor-craft appx. $20-40 \mathrm{~km} / \mathrm{h}$ ) and having the advantage of far more flight endurance between $60 \mathrm{~min}$ and $120 \mathrm{~min}$ (rotor-craft appx. 20 - $40 \mathrm{~min}$ ) the question arises if survey accuracy and image quality decrease when operating at higher velocities. Survey accuracy is determined using the aforementioned geodetic-photogrammetric test field and image quality in terms of spatial resolution according to the upcom-

\footnotetext{
*Corresponding author
}

ing German standard DIN 18740-8 "Photogrammetric products Part 8: Requirements for image quality (quality of optical remote sensing data)“.

Therefore, the used prototype camera system will be described in section 2. A short review of the test field and terrestrial geodetic surveying is given in section 3 . The planned and executed campaign with its underlying preliminary considerations is described in section 4. A short review on image quality and spatial resolution measurement is given in section 5 followed by a theoretical description of motion smear and a benchmark procedure to compare sensor-lens combinations under static (laboratory) and kinematic (operating) conditions in the same section. Related results for survey accuracy and spatial resolution are presented in section 6 followed by several conclusions in section 7 .

\section{CAMERA SYSTEM}

Due to national law policies the maximum take-off weight (MTOW) is often restricted which also leads to limitations for the payloads. Using carriers with an MTOW less $5 \mathrm{~kg}$ is quite popular in Germany (BMVI, 2016) which was the starting point to develop a lightweight and metric aerial camera system (Kraft et al., 2016b) with the intention to verify the system using traditional photogrammetric evaluation procedures (Kraft et al., 2016a). This investigation confirmed that the prototype is a metric camera system with long term stable interior orientation.

Based on this metric camera system the first prototype of the drone-based real-time mapping camera was developed in 2018 (see figure 1). The system incorporates an industrial camera, a dual-frequency GNSS receiver including inertial-aided attitude processing (INS), and an embedded computer. The camera head consists of a 16 MPix CCD sensor (ON Semi-conductor 

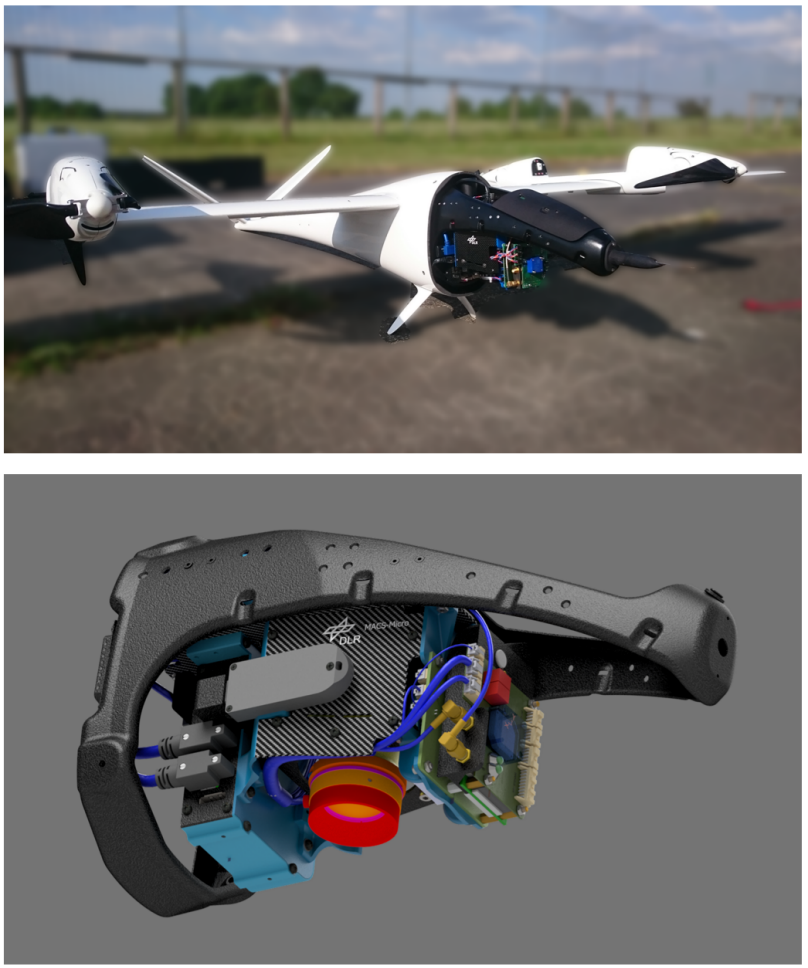

Figure 1. (top) Prototype of the camera system mounted to carrier, (bottom) CAD-model of the camera system

KAI-16070 with Bayer pattern) and an industrial F-Mount lens (Schneider Kreuznach Xenon-Emerald 2.2/50). The aperture is set to 4.0 and the focus is fixed to the hyperfocal distance. Exterior orientation calculation is based on a dual-antenna GNSS receiver (Novatel OEM7720) in combination with an industrial grade MEMS-IMU (Epson G320N). The dual-antenna set-up is used to determine true-heading independently from INS. This improves the orientation accuracy, in particular when movement direction and heading do not correlate due to cross-wind. Depending on flight trajectory, differences of up to 10 degrees have been observed. Additionally the dual-antenna system allows for very fast attitude initialization already on ground without aircraft movement. The distance (basis) between both GNSS antennas (mounted in the front and tail) is $0.95 \mathrm{~m}$. The GNSS receiver continuously estimates position and attitude. The end-of-exposure signal is signaled to the GNSS receiver.

Thus, every image is assigned with precisely measured time, position and orientation. Considering the interior camera orientation (long-term stable) direct geo-referencing can be applied. Due to continuous synchronization of all subsystems, each image can be time-stamped with a precision better than $1 \mu \mathrm{s}$. Time synchronization, image acquisition and real-time image processing is done by the embedded computing unit. This computer is powered by a Quad Core Processor (Intel Atom E3950) with 8 GB RAM and runs a Linux operating system. In this configuration the system allows to capture up to 4 raw images per second which can be stored on an removable storage device. The camera system is shown in figure 1 (bottom). The weight is $1.4 \mathrm{~kg}$ (including embedded PC, camera, IMU, GNSS receiver, GNSS antenna, power management and structure) and the dimensions are $10 \times 14 \times 20 \mathrm{~cm}^{3}$.

A fixed-wing drone (see figure 1, top) is used as carrier providing a flight time of approximately 90 minutes at cruise speeds between $60 \mathrm{~km} / \mathrm{h}$ and $90 \mathrm{~km} / \mathrm{h}$. Thus, the carrier is capable to travel a distance of up to $105 \mathrm{~km}$ per battery charge. It is specified with MTOW of $14 \mathrm{~kg}$ including a payload of up to $2 \mathrm{~kg}$ and has a wingspan of $3.5 \mathrm{~m}$. It can operate at wind speeds of up to $8 \mathrm{~m} / \mathrm{s}$ and temperatures between $0^{\circ} \mathrm{C}$ and $35^{\circ} \mathrm{C}$. While its typical flight operation altitude is in the range between $100 \mathrm{~m}$ and $300 \mathrm{~m}$ above ground level, it is capable of operating at altitudes up to $3,000 \mathrm{~m}$ above sea level.

The operational range is only limited by the maximum flight time because the autopilot systems allows fully automated flights beyond visual line of sight (BVLOS). This requires a predefined flight plan with terrain follow mode for security reasons. The drone is equipped with a conventional command and control link as well as an additional mobile network radio for BVLOS operation. For safety reasons this carrier is equipped with position lights and an integrated automatic dependent surveillance broadcast (ADS-B) transceiver.

\section{TEST FIELD AND TERRESTRIAL GEODETIC SURVEYING}

The area of Zeche Zollern, also used as test field in ISPRS Benchmarks (Nex et al., 2015, Przybilla et al., 2019), covers almost the entire area of the open space museum (see figure 2) and has an extent of $320 \mathrm{~m} \mathrm{x} 220 \mathrm{~m}$. The highest vertical point is given by the approx. $40 \mathrm{~m}$ high pitheads. It consists of 45 rotor-shaped signaled ground points and 3 Siemens-stars, which are used for image quality analysis.

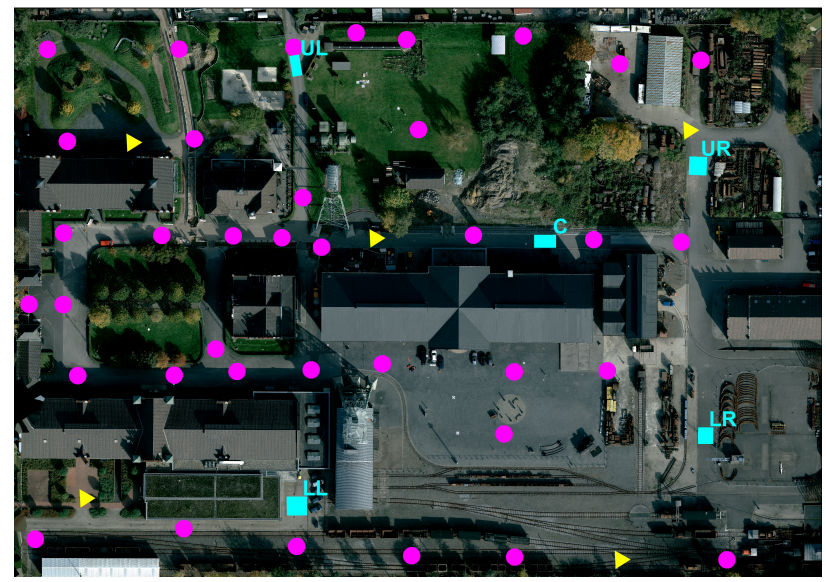

Figure 2. Orthophoto of test field Zeche Zollern with GCP (yellow triangle)/Check point (magenta circle) distribution and areas of surface analysis (cyan rectangle)

The network measurements were carried out using tachymeters, a precision level and an RTK-GNSS system (Przybilla et al., 2018): "To determine the coordinates of the control points in ETRS89 system, GNSS RTK measurements using the SAPOS HEPS service have been obtained. The UTM coordinates and ellipsoidal heights of these measurements served as data points for the subsequent 3D network adjustment. The ellipsoidal heights of the GNSS measurements were additionally transformed with the quasi-geoid heights of the GCG2016 to obtain normal heights of the DHHN2016. They were used as connecting heights in the following adjustment of the leveling measurements. The standard deviations of the adjusted normal heights in the system DHHN2016 (NHN) are in a range of $1 \mathrm{~mm}$ to $3 \mathrm{~mm}$ and their relative accuracy is better than $1 \mathrm{~mm}$. For the network measurements 


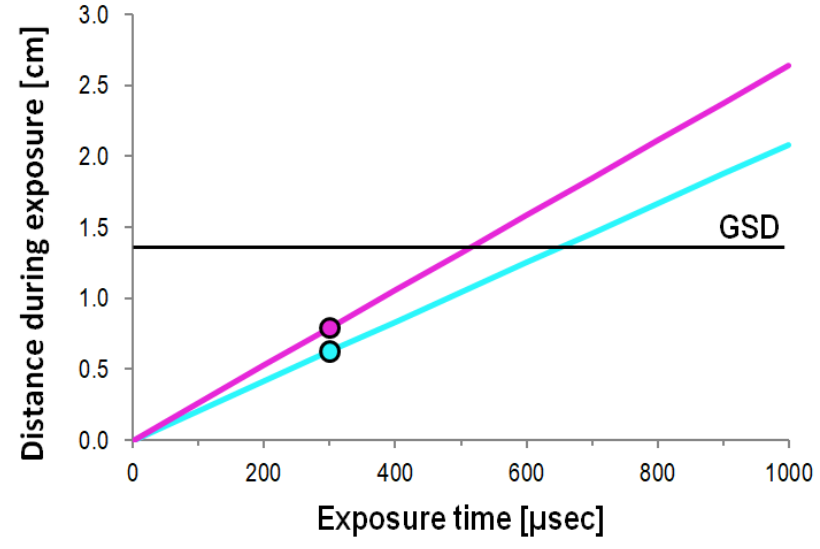

Figure 3. Relation between distance during exposure at $75 \mathrm{~km} / \mathrm{h}$ (cyan) and $95 \mathrm{~km} / \mathrm{h}$ (magenta) at different exposure times.

a standard deviation of a single coordinate for the 15 common tachymeter survey stations, which could be measured in forced centering, was $1.2 \mathrm{~mm}$. The corresponding value for the ground control points (GCP) was $2.5 \mathrm{~mm} . ”$.

\section{FLIGHT CAMPAIGN}

The altitude above ground level of the image flight was $95 \mathrm{~m}$ resulting in ground sample distance (GSD) of approximately $1.4 \mathrm{~cm}$. Planned flight speed was $75 \mathrm{~km} / \mathrm{h}$. In total, 340 images of 9 alternating flight strips with an overlap of $80 \%$ in track and $60 \%$ across track have been used for evaluation. In addition one strip was planned with maximum speed of $95 \mathrm{~km} / \mathrm{h}$.

As already mentioned, the exposure time is important in terms of avoiding image blur due to over-exposure. Figure 3 shows the linear relation between exposure time and flight speed at the planned altitude and GSD. With a planned exposure time of $300 \mu \mathrm{sec}$, the flown distance during exposure is $6 \mathrm{~mm}$ at $75 \mathrm{~km} / \mathrm{h}$ and $8 \mathrm{~mm}$ at $95 \mathrm{~km} / \mathrm{h}$ and thus below GSD of $1.4 \mathrm{~cm}$. Basically, flight planning follows the proposed procedure of (Wenzel et al., 2013) for image data acquisition. Image overlap is crucial for the quality and completeness of the later derived point cloud or surface model from these images. This is just one important aspect, however, a wide field of view causes objects to be imaged tilted (distorted) in the overlapping image area and occlusions might occur. Further point correspondences (features) are more difficult or impossible to find because of low image similarity. In addition to the overlap specifications, maximum intersection angle between identical image features must be considered. For high image similarity angular differences should be kept below 15 degrees (Wohlfeil et al., 2013). The range of intersection angles for this camera system and planned flight was between 5 degree and 24 degree in track and maximum 15 degree across track to adjacent strips.

Table 1. Estimated height precision for planned flight

\begin{tabular}{c|r|c}
\hline Overlap [\%] & $\mathrm{B}[\mathrm{m}]$ & $\Delta_{Z}[\mathrm{~cm}]$ \\
\hline 80 & 9.1 & 2.9 \\
60 & 18.2 & 1.5 \\
40 & 27.4 & 1.0 \\
20 & 36.5 & 0.7
\end{tabular}

If the automatic measurement accuracy $\Delta_{B}$ is defensively approximated with $1 / 5$ Pixel, based on planned base lengths $B$ between two stereo images at height above ground level $Z$ of $95 \mathrm{~m}$, height accuracy $\Delta_{Z}$ for a single stereo measurement in strip can be estimated (see table 1) according to (Kraus, 2007):

$$
\Delta_{Z}=\frac{Z^{2}}{c_{K} B} \Delta_{B}
$$

Horizontal accuracy $\Delta_{X}, \Delta_{Y}$ is calculated independently (Kraus, 2007) with

$$
\begin{gathered}
\Delta_{X}=\Delta_{Y}=\frac{Z}{c_{K}} \Delta_{B} \\
\Delta_{X}=\Delta_{Y}=\frac{95 \mathrm{~m}}{50 \mathrm{~mm}} \frac{7.4 \mu \mathrm{m}}{5}=0.3 \mathrm{~cm}
\end{gathered}
$$

Thus, the expected horizontal point accuracy $(0.3 \mathrm{~cm})$ of stereo images with this camera and flight plan is in an approximated range of $1 / 4 \mathrm{GSD}$ and the vertical point accuracy $(1.5 \mathrm{~cm})$ in the range around $1 \mathrm{GSD}$.

\section{IMAGE QUALITY}

Image quality of a sensor system is affected by multiple factors and directly influences perceptible detail in aerial images. Light rays which are being reflected by an object and detected by a camera sensor partially traverse the atmosphere and loose some of their energy due to diffusion and absorption. In drone applications this part could be considered very small and won't be discussed further here.

Next the light passes a (complex) lens system where an aperture is integrated and limits the effective solid angles for every ray. As a consequence the lens-aperture directly affects the amount of light which in turn determines the amount of photons that reach the sensor plane and contribute to the imaging process. The smaller the aperture is chosen the more diffraction of light limits a sharp optical imaging. On the other hand, if the aperture is chosen too large spherical and chromatic aberrations gain influence. The amount of photons passing through the lens system and reaching the sensor at a distinct time frame directly influences the exposure time needed to create an equivalent sensor signal. In aerial photogrammetry the exposure time however affects a sharp optical imaging in terms of motion blur that is a result of the system's change of location / movement whilst the sensor is exposed. This change of location can be compensated actively and several remote sensing systems offer some techniques. But almost all systems for drones are not equipped with according solutions as additional parts increase total weight limiting flight endurance and operation time.

Another interfering aspect is the gain of shading (or inverse the luminous intensity decrease) starting from the principle point to image corners. This effect is often described as vignetting and is caused by the lens-system itself and by the integrated aperture. Vignetting can be measured and corrected as an image processing step whilst determine the Photo Response Non-Uniformity (PRNU) (Wg EMVA, 2016).

After the light rays passed the lens-system they hit the sensor surface. That part of the camera system creates a digital interpretable signal and directly depends on the amount of collected photons during the exposure time window. The quality of that signal is affected by several electronic components (e.g. sensor 
read-out electronic, analog-digital converter). A measure of this quality is the signal-to-noise-ratio (SNR). The SNR also is characterized by a) the ambient noise level that unavoidably occurs when a semi-conductor is connected to its supply voltage and $b$ ) to the photo-effective area of each sensor element (pixel). The larger the effective area the more photons contribute to the signal assuming identical time frames and therefore increase the signal. Electronic ambient noise can be determined pixel by pixel as part of the Dark Signal Non-Uniformity (DSNU) (Wg EMVA, 2016).

\subsection{Spatial Resolution Determination}

Spatial resolution is an essential parameter of imaging systems (Meißner et al., 2018) as it defines a measure of imaged detail for every image taken by a sensor-lens configuration. Therefore resolution estimation is important to quantify the potential of aerial camera systems. Spatial resolution as an image quality parameter is part of the new upcoming German standard DIN 18740-8 "Photogrammetric products - Part 8: Requirements for image quality (quality of optical remote sensing data)“.

Spatial resolution can be defined mathematically as follows: A point-like input signal $U\left(x^{\prime}, y^{\prime}\right)$ with object space coordinates $x^{\prime}$ and $y^{\prime}$ will be spread (or smeared) due to non-ideal imaging properties (Jahn and Reulke, 1995) and creates an output signal $V(x, y)$ with image coordinates $x$ and $y$ :

$$
V(x, y)=\iint d x^{\prime} d y^{\prime} H\left(x, y, x^{\prime}, y^{\prime}\right) U\left(x^{\prime}, y^{\prime}\right)
$$

The spread (or smeared) output signal depends on the system impulse response $H(r)=H\left(x, y, x^{\prime}, y^{\prime}\right)$ with $r=$ $\sqrt{\left(x-x^{\prime}\right)^{2}+\left(y-y^{\prime}\right)^{2}}$ which is therefore called point spread function (PSF) (Williams and Becklund, 1989, Jahn and Reulke, 1995).

Furthermore, sharpness as an image property can be characterized by the modulation transfer function (MTF) $\tilde{H}(k)$ which is the spatial frequency response of an imaging system to a given illumination. "High spatial frequencies correspond to fine image detail. The more extended the response, the finer the detail - the sharper the image." (Mix, 2005) and is equal to Fourier transform of PSF $H(r)$ :

$$
H(r) \circ \bullet \tilde{H}(k)
$$

The effective resolution of an imaging device can be determined in different ways. A classic approach is the use of well-known test charts (e.g. USAF resolution test chart with groups of bars) (USAF, 1959). There, the (subjectively) identified image resolution corresponds to that distance where the smallest group is still discriminable. This is very similar to the Rayleigh resolution limit (Rayleigh, 1874). There, the response of an imaging system when illuminated with a point light source is defined and approximated by a sine cardinal function. Further, Rayleigh postulated the resolution limit as the minimal distance between the two sources where they are still discriminable. Using the definition that point light sources are approximated as sine cardinal functions the resolution limit is reached if the first maximum position of one function is identical to the first minimum of the other function.

Besides subjective components included in this process the function values (ground resolved distance - GRD) are discrete instead of continuous, depending on resolution steps between groups of bars.

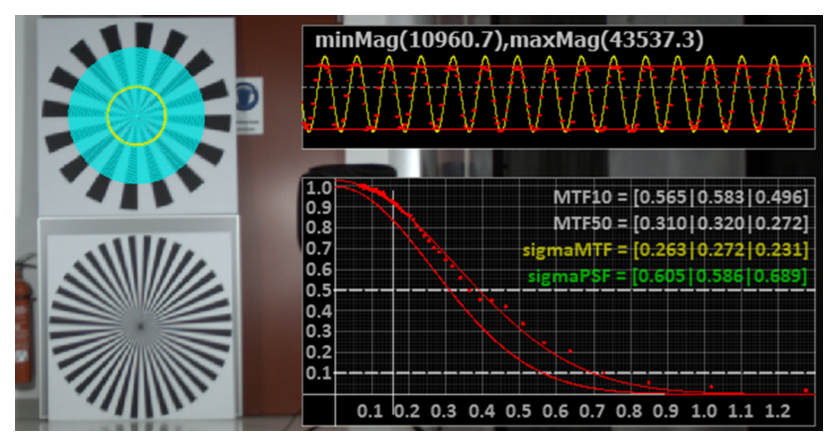

Figure 4. Designated test pattern Siemens-star (left), radial modulation analysis (top-right), resulting MTF and PSF (bottom-right).

To reduce subjective influence with bar charts during the determination process and to convert discrete function values to continuous some approaches use signal processing techniques to calculate effective image resolution. The method described by (Reulke et al., 2004, Reulke et al., 2006) is one of the latter approaches. There, the contrast transfer function (CTF) and subsequently MTF is calculated for images with a designated test pattern (e.g. Siemens-star - see figure 4). According to the above mentioned approaches the smallest recognizable detail or "the resolution limit is reached if the distance between two points leads to a certain contrast in image intensity between the two maxima." Using a priori knowledge of the original scene (well-known Siemensstar target) CTF, MTF and PSF can be approximated e.g. by a Gaussian shape function (Honkavaara et al., 2006) or polynomial function.

Coordinate axis $X$ for CTF and MTF is the spatial frequency $k$ (eq. 6) and is calculated as the quotient of target frequency $k_{s}$ divided by current scan radius $r$ multiplied by $\pi$. Target frequency $k_{s}$ is constant and equivalent to the number of blackwhite Siemens-star segments. Related (initially discrete) values for contrast transfer function $C_{d}(k)$ are derived using intensity maximum $I_{\max }$ and minimum $I_{\min }$ for every scanned circle (eq. 7). Simultaneously the function value is normalized to contrast level $C_{0}$ at spatial frequency equal to 0 (infinite radius). Continuous function values $C$ are derived by either fitting a Gaussian function into discrete input data or e.g. a fifth order polynomial. According to (Coltman, 1954) the obtained CTF describes the system response to a square wave input while MTF is the system response to a sine wave input. The proposed solution is a normalization with $\frac{\pi}{4}$ followed by series expansion using odd frequency multiples (eq. 8).

$$
\begin{gathered}
k=\frac{k_{s}}{\pi r} \\
C_{d}(k)=\frac{I_{\max }(k)-I_{\min }(k)}{I_{\max }(k)+I_{\min }(k)} * \frac{1}{C_{0}} \\
\tilde{H}(k)=\frac{\pi}{4}\left[C(k)+\frac{C(3 \cdot k)}{3}+\frac{C(5 \cdot k)}{5}+\ldots\right]
\end{gathered}
$$

There are several criteria specifying resolving power of camera systems. The parameter $\sigma$ (standard deviation) of the PSF (assuming Gaussian-shape) is one criterion. It directly relates to image space and can be seen as objective measure to compare different camera performances. Another criterion is the width of PSF at half the height of its maximum (full width half maximum - FWHM).

The value for MTF at $10 \%$ modulation contrast often is referred 


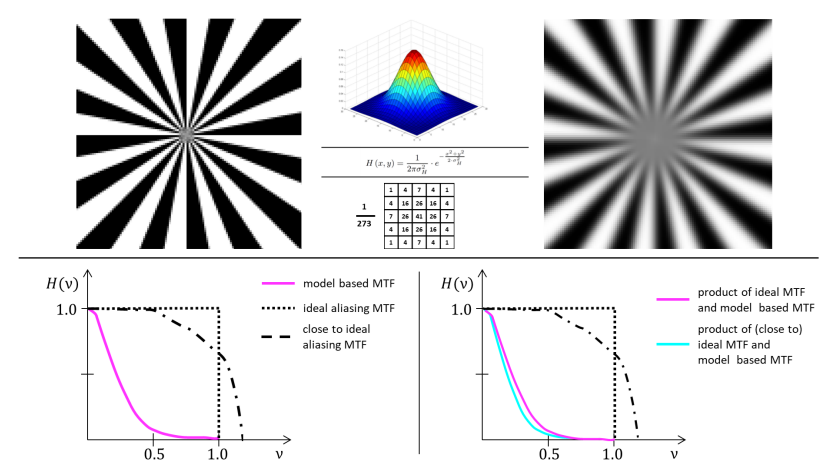

Figure 5. Original image (upper left) continuous and discrete Gaussian PSF convolution kernel (upper mid) and convolution result (upper right), ideal aliasing MTF, close to ideal aliasing MTF and model-based MTF (lower left), related products in frequency domain (lower right)

to as resolution limit or cut-off frequency of MTF $\tilde{H}(k)=0.10$ at spatial frequency $k_{M T F 10}$ where it's reciprocal $H(r)$ (PSF) corresponds to the least resolved scale in image domain. This scale factor multiplied by nominal ground sample distance then delivers the least resolved distance and is named ground resolved distance (GRD) (Kharfi et al., 2012, Artmann and Wueller, 2012, Valenzuela and Reyes, 2019, Nakamura, 2016).

\subsection{Motion smear}

Sensor motion of aerial imaging systems can be described as sensor-rotation with three DOF (roll, pitch, yaw), sensortranslation with three DOF ( $X Y, Z$, e.g. world coordinates) or as both motions at the same time. An optical sensor under motion during image acquisition not only collects photons of the static pixel-footprint projected onto the observed surface but of the extended footprint along the projected line of movement. Then, the input signal $U\left(x^{\prime}, y^{\prime}\right)$ (eq. 4) can be described as an integral along the projected line of movement in object space:

$$
U\left(x^{\prime}, y^{\prime}\right)=\int U_{\sigma}\left(x^{\prime}, y^{\prime}, m\right) d m
$$

Where $U_{\sigma}\left(x^{\prime}, y^{\prime}, m\right)$ is the input signal at every projected position depending on motion $m$ (6 DOF) during the exposure time window.

During that window motion induces smear which directly affects the overall PSF $H(r)$ (eq. 4). With increasing motion (e.g. 1Dtranslation in flight direction of the drone at high velocity) the PSF will be smeared along the projected motion. That hypothesis can be investigated empirically by a simulation. The sequence is to apply predefined modulation (MTF) or spread parameters (PSF) to an ideal representation of resolving patterns (see fig. 5). That can be done by forming a convolution of mathematical-ideal image-intensity values of an image $(I)$, a Gaussian-shape model PSF $\left(H_{m}\right)$ and a mathematical-ideal aliasing PSF $\left(H_{s}\right)$. Simulated PSF $\left(H_{\text {sim }}\right)$ then can be formulated as follows (Meißner et al., 2020):

$$
H_{\text {sim }}(\rho)=I(\rho) * H_{m}(\rho) * H_{s}(\rho)
$$

Convolution of image-intensity values $I(\rho)$ and an increasingly smeared kernel (initial) Gaussian-shape (see figure 6, top) then delivers a more and more stretched version of corresponding

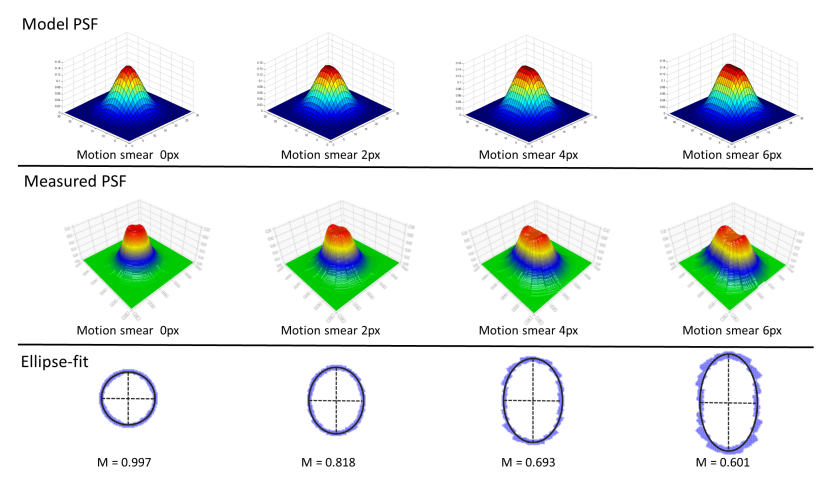

Figure 6. (top) Model convolution-kernel with rising influence of motion from 0 to 6 pixel, (middle) measured PSF of images convoluted with model-kernels, (bottom) corresponding ellipse fit for 2D-MTF10 and relation $M$ of semi-minor ellipse-axis $e_{s}$ and semi-major ellipse-axis $e_{l}$

measured PSF $H(r)$ (see figure 6, middle). Measured PSF of a motionless kernel is circle-shaped and becomes increasingly elliptic-shaped when motion gains influence (see figure 6, bottom). Therefore, the length between semi-minor ellipse-axis $e_{s}$ and semi-major ellipse-axis $e_{l}$ can be used as measure $M$ [\%] of induced motion:

$$
M=\frac{e_{s}}{e_{l}}
$$

Then, circle-shaped 2D-PSFs and corresponding ellipse fit will deliver values for $M$ close to 1.00 and decreasing values for increasing elliptic-shape of measured 2D-PSF (see figure 6, bottom). This measure will be used to evaluate motion smear of the camera system (see section 2) designed for fixed-wing applications. Results are given in section 6.2.1.

\subsection{Sensor Validation}

Usually, spatial resolution of a sensor-lens combination under laboratory conditions will be significantly better than under operating and thus kinematic conditions due to aforementioned vibrations and motion (6 DOF) of the carrier. It is therefore beneficial to determine and compare spatial resolution in both cases.

5.3.1 Laboratory measurement The benchmark procedure to determine spatial resolution parameters for a specific sensorlens combination under laboratory conditions is defined as follows (Meißner et al., 2017). In order to guarantee a repeatable measurement procedure with identical controlled light conditions and to prevent extraneous light a sufficiently large basement hall has been identified (see figure 7). The GSD in this benchmark (according to focal length and sensor pixel-size) has been set to $1.0 \mathrm{~cm}$ to address the aforementioned fields of application including their resolution requirements. Usually, resolving power is changing across the field of view. In order to analyze this effect multiple images have been taken and the resolution target is imaged at different locations in image space (e.g. image center - image half field - image corner). For every image the MTF10 (see section 5.1) has been calculated. This should guarantee the genuine system response from object space to sensor for the expected field of applications. Results are presented in section 6.2.2. 

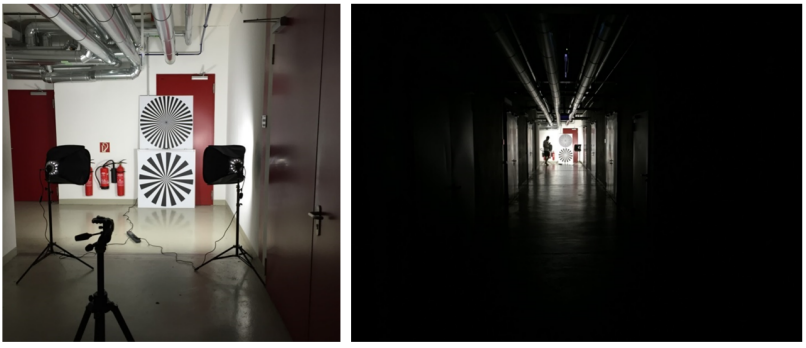

Figure 7. Controlled light conditions (left), no extraneous light (right)

5.3.2 In-field measurement As mentioned before, spatial resolution parameter of a camera system in motion is expected to be lower than under (static) laboratory conditions. Main reason is the sensor movement (6 DOF) through 3D-space but with additional influence of the carrier-unit's (micro-)vibrations under operating conditions.

Prepared and correctly executed flight plans, introduced in section 4 with overlaps of $80 \%$ in track and $60 \%$ across track ensure the spatial resolution test target (Siemens-star) to be in at least 15 images. Simultaneously the test target is imaged at different image locations. Similar to the benchmark procedure under static conditions (see section 5.3.1) images containing the Siemens-star at center-, half-field and corner-position have been selected for measurement and comparison. Results are given in section 6.2.2.

\section{RESULTS}

\subsection{Test Field Validation}

The data processing chain consists of aerial triangulation for all images and subsequent dense image matching for surface reconstruction. Image orientation as the result of aerial triangulation has been calculated including 4 GCP in the corners and 1 GCP in the test field center. The remaining 40 points were used as checkpoints $(\mathrm{CP})$ especially to verify relative accuracies of the bundle block (see figure 2). After bundle block adjustment the checkpoints' horizontal RMS was $4.0 \mathrm{~mm}$ and vertical RMS $6.0 \mathrm{~mm}$ as shown in figure 8 . Balanced distribution of 40 independent checkpoints allows to conclude that it is possible to derive an object model (with no model distortion) which is accurate having a 3D-error standard deviation of approximately $7.2 \mathrm{~mm}$.
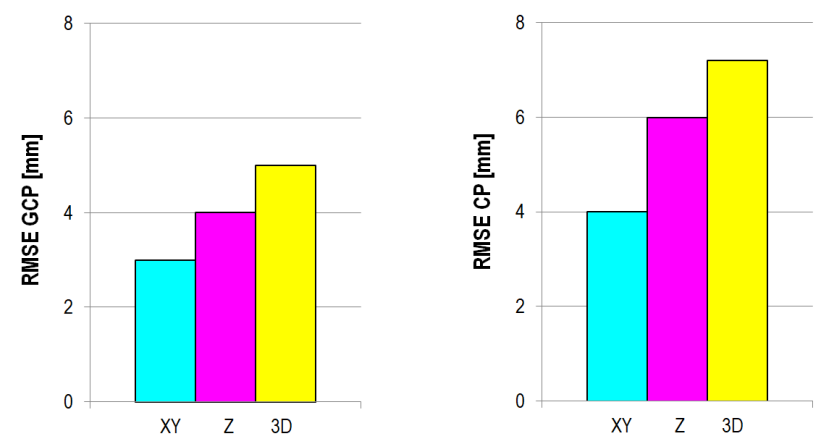

Figure 8. RMSE of 5 GCP (left) and $40 \mathrm{CP}$ (right) after bundle block adjustment

While this procedure only considers checked single points and its absolute accuracy before the automatically step of 3D point
Table 2. Standard deviation of unfiltered point cloud from equalization plane

\begin{tabular}{l|c|c}
\hline AreaID & Count & StdDev $[\mathrm{cm}]$ \\
\hline UpperLeft & 2024291 & 1.6 \\
UpperRight & 1971705 & 1.3 \\
LowerRight & 2052980 & 2.2 \\
LowerLeft & 2125558 & 1.8 \\
Center & 1958797 & 1.2
\end{tabular}

cloud generation, the point cloud obtained by multi-view dense image matching was used to investigate if the relative accuracy of 3D points corresponds to preliminary considerations for typical smooth and planar surfaces. Therefore, noise of the unfiltered point cloud was examined and quantified at five different surfaces (see figure 2). Unfiltered point cloud entails the raw output of all possible stereo matches. Well distributed areas have been selected where dense image matching seems expedient, for example where noise is not caused by patterns being too homogeneous. The average number of points was around 2 million per examined surface. The results are represented in table 2 .

As estimated for the flight campaign (see section 4), measurements deviate in the range of $1.20 \mathrm{~cm}$ and $2.20 \mathrm{~cm}$. From a photogrammetric point of view, it can therefore be concluded that the camera works in the range of planned and predicted accuracy even at high velocities.

\subsection{Spatial Resolution}

This sub-section is divided into two parts. The first part presents the results for motion smear in drone images (see section 5.2) followed by sensor validation under static and kinematic conditions (see section 5.3).

6.2.1 Motion Smear A general comparison of aerial images with and without dominant motion smear is given in figure 9 . The image in the upper row suffers from sharpness-loss due to motion smear. This becomes clear when looking at the grade of deformation of 2D-PSF. Quotient $M$ in this case (see eq. 11) is 0.680 . The image in the lower row does not suffer from dominant motion smear and related Quotient $M$ in that case is 0.967 .
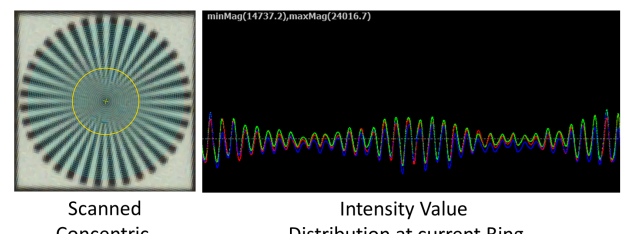

Rings Distribution at current Ring

$$
\text { (yellow) }
$$
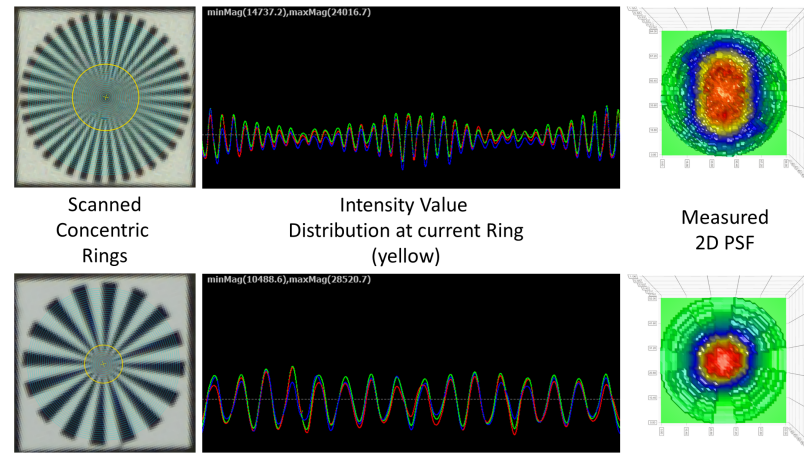

Figure 9. (top) Drone image with dominant motion smear, (bottom) drone image without dominant motion smear

The Siemens-star test target has been imaged at 15 different locations in image space by maintaining predefined flight plan (see section 4). Using the software-tool for spatial resolution (see section 5.1) the 2D-PSF and corresponding quotient $M$ (relation of semi-minor and semi-major ellipse axis) have been calculated 
Table 3. Quotient M of semi-minor and semi-major ellipse axis for images under kinematic conditions.

\begin{tabular}{c|c}
\hline Image ID & Quotient $M\left(e_{s}, e_{l}\right)$ \\
\hline 00583.tif & 0.910 \\
00584.tif & 0.893 \\
00585.tif & 0.899 \\
00586.tif & 0.974 \\
00587.tif & 0.943 \\
00588.tif & 0.900 \\
00656.tif & 0.904 \\
00657.tif & 0.951 \\
00658.tif & 0.961 \\
00659.tif & 0.876 \\
00660.tif & 0.868 \\
02094.tif & 0.887 \\
02096.tif & 0.928 \\
02097.tif & 0.889 \\
02208.tif & 0.894 \\
\hline Mean & $\mathbf{0 . 9 1 2}$ \\
\hline
\end{tabular}

for every position. The results are given in table 3 . Mean value for $M=0.912$ reveals that percentage of length-difference between semi-minor and semi-major axis is small. Furthermore, it strongly indicates that 2D-PSF is rather circle-shaped and leads to the conclusion that there is no dominant motion smear present for this particular flight campaign.

6.2.2 Sensor Validation As explained in section 5.3 spatial resolution parameters for static (laboratory) conditions are expected to be better than under kinematic (operating) conditions due to motion caused by carrier-movement and -vibrations. Further, spatial resolution is expected to be better when imaged in center than in image corners. To investigate both issues, images under static conditions containing the Siemens-star at center-, half-field and corner-position have been selected for measurement. The same selection has been made for kinematic conditions. For every image the value for MTF at $10 \%$ contrast level (resolution limit, see section 5.1) has been calculated and serves as measure to conduct the analysis. Results for both aspects are given in figure 10. As expected, resolution limit (MTF10) declines from image-center via image-half-field to image-corner for both static and kinematic conditions. This deterioration is mainly caused by chromatic and spherical aberrations induced by the lens-system. Furthermore, the mean difference between operating and static conditions is $23.6 \%$ (center $20.9 \%$, half-field $22.7 \%$, corner $27.2 \%$ ). Considering very high image performance under laboratory conditions (around 1.00 line/pixel and thus near Nyquist-limit) a reduction of $23 \%$ of resolution under operating conditions still can be considered satisfactory.

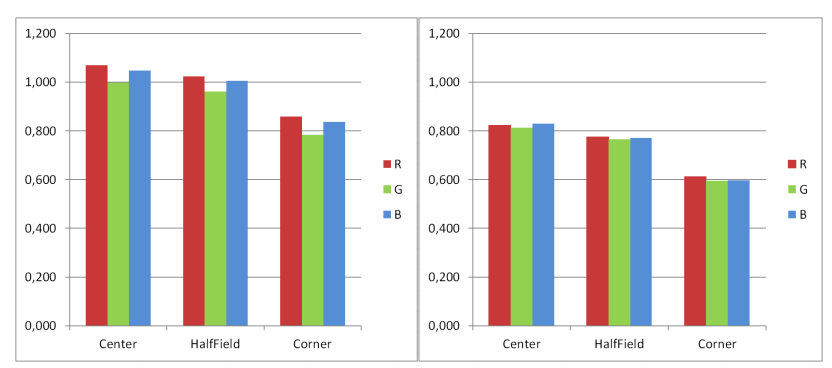

Figure 10. Results for resolving power measurements [line/pixel] under static (left) and kinematic (right) conditions.

\section{SUMMARY AND CONCLUSION}

An evaluation of a camera system prototype for fixed-wing drones has been presented. Focus of this investigation was to find out if higher velocities during image acquisition has any influence on photogrammetric survey and image quality itself in comparison to common rotor-craft drones.

Several preliminary considerations have been described such as general expectation of survey accuracy and the relation between exposure-time and carrier velocity. Furthermore, a measure for motion smear has been introduced and evaluated with simulated motion-PSF.

Survey accuracy has been determined using a geodeticphotogrammetric test field. Having included 5 GCP and $40 \mathrm{CP}$ the standard deviation of 3D-error is approximately $7.2 \mathrm{~mm}$ and compared to other camera systems mentioned in (Przybilla et al., 2019) similar or even slightly better.

Presence of motion smear has been investigated with a software tool for spatial resolution determination. It has been shown that obtained aerial images and corresponding image quality do not suffer from motion smear. The mean loss of resolving power under operating conditions (compared to laboratory conditions) of approximately $23.6 \%$ is satisfactory and expected.

It can therefore be concluded that the presented camera system and carrier deliver very reliable and high precision results in terms of survey accuracy and spatial resolution. The presented fixed-wing system entails the advantage of much higher flight endurance while maintaining survey accuracy of slower carriers without sacrificing image quality.

\section{ACKNOWLEDGEMENTS}

The authors would like to thank Prof. Dr. Heinz-Jürgen Przybilla, Prof. Dr. Manfred Bäumker and their students of Bochum University of Applied Sciences (HSBO) for preparing and carrying out the complex network measurement and campaign at Zeche Zollern open space museum.

The current software version to determine spatial resolution of imagery in a standardized way according to DIN 18740-8 together with the designated Siemens-star template can be made available to interested readers. Please contact the main author of this paper.

\section{REFERENCES}

Artmann, U. and Wueller, D., 2012. Improving texture loss measurement: spatial frequency response based on a colored target. In: Image Quality and System Performance IX, Vol. 8293, International Society for Optics and Photonics, p. 829305.

Bakuła, K., Ostrowski, W., Pilarska, M., Szender, M. and Kurczyński, Z., 2018. Evaluation and calibration of fixed-wing uav mobile mapping system equipped with lidar and optical sensors. International Archives of the Photogrammetry, Remote Sensing \& Spatial Information Sciences.

BMVI, 2016. Kurzinformation über die Nutzung von unbemannten Luftfahrtsystemen. Bundesministerium für Verkehr und digitale Infrastruktur.

Coltman, J. W., 1954. The specification of imaging properties by response to a sine wave input. JOSA 44(6), pp. 468-471. 
Hein, D., Kraft, T., Brauchle, J. and Berger, R., 2019. Integrated uav-based real-time mapping for security applications. ISPRS International Journal of Geo-Information 8(5), pp. 219.

Honkavaara, E., Jaakkola, J., Markelin, L. and Becker, S., 2006. Evaluation of resolving power and MTF of DMC. International Archives of Photogrammetry, Remote Sensing and Spatial Information Sciences.

Hruska, R., Mitchell, J., Anderson, M. and Glenn, N. F., 2012. Radiometric and geometric analysis of hyperspectral imagery acquired from an unmanned aerial vehicle. Remote Sensing 4(9), pp. 2736-2752.

Jahn, H. and Reulke, R., 1995. Systemtheoretische Grundlagen optoelektronischer Sensoren. Akademie Verlag.

Khan, S., Aragão, L. and Iriarte, J., 2017. A uav-lidar system to map amazonian rainforest and its ancient landscape transformations. International journal of remote sensing 38(8-10), pp. 2313-2330.

Kharfi, F., Denden, O., Bourenane, A., Bitam, T. and Ali, A., 2012. Spatial resolution limit study of a ccd camera and scintillator based neutron imaging system according to $\mathrm{mtf}$ determination and analysis. Applied Radiation and Isotopes 70(1), pp. 162-166.

Kraft, T., Geßner, M., Meißner, H., Cramer, M., Przybilla, H.J. and Gerke, M., 2016a. Evaluation of a metric camera system tailored for high precision UAV applications. In: International Society for Photogrammetry and Remote Sensing, Volume XLI$\mathrm{B} 1,2016$.

Kraft, T., Geßner, M., Meißner, H., Przybilla, H.-J. and Gerke, M., 2016b. Introduction of a photogrammetric camera system for RPAS with highly accurate GNSS/IMU information for standardized workflows. In: J. Skaloud and I. Colomina (eds), EuroCOW 2016, the European Calibration and Orientation Workshop (Volume XL-3/W4), pp. 71-75.

Kraus, K., 2007. Photogrammetry Geometry from Images and Laser Scans. Vol. 2nd Edition, Walter de Gruyter.

Meißner, H., Cramer, M. and Piltz, B., 2017. Benchmarking the optical resolving power of UAV based camera systems. In: UAVg 2017, The International Archives of Photogrammetry, Remote Sensing and Spatial Information Sciences, 42., p. 243.

Meißner, H., Cramer, M. and Reulke, R., 2018. Towards standardized evaluation of image quality for airborne camera systems. ISPRS - International Archives of the Photogrammetry, Remote Sensing and Spatial Information Sciences XLII-1, pp. 295300.

Meißner, H., Cramer, M. and Reulke, R., 2020. Evaluation of structures and methods for resolution determination of remote sensing sensors. In: J. J. Dabrowski, A. Rahman and M. Paul (eds), Image and Video Technology, Springer International Publishing, Cham, pp. 59-69.

Mix, P. E., 2005. Introduction to Nondestructive Testing: A Training Guide. JOHN WILEY \& SONS INC.

Nakamura, J., 2016. Image sensors and signal processing for digital still cameras. CRC press.

Nex, F., Gerke, M., Remondino, F., Przybilla, H.-J., Bäumker, M. and Zurhorst, A., 2015. ISPRS benchmark for multi-platform photogrammetry. ISPRS Annals of Photogrammetry, Remote Sensing and Spatial Information Sciences II-3/W4, pp. 135-142.

Przybilla, H.-J., Bäumker, M. and Vieten, J., 2018. Das UAVTestfeld Zeche Zollern in Dortmund. In: Proceedings of the DVW, pp. 61-80.
Przybilla, H.-J., Gerke, M., Dikhoff, I. and Ghassoun, Y., 2019. Investigations on the geometric quality of cameras for UAV applications using the high precision UAV test field zollern colliery. ISPRS - International Archives of the Photogrammetry, Remote Sensing and Spatial Information Sciences XLII-2/W13, pp. 531538.

Rayleigh, L., 1874. XII. On the manufacture and theory of diffraction-gratings. The London, Edinburgh, and Dublin Philosophical Magazine and Journal of Science 47(310), pp. 81-93.

Reulke, R., Becker, S., Haala, N. and Tempelmann, U., 2006. Determination and improvement of spatial resolution of the CCDline-scanner system ADS40. ISPRS Journal of Photogrammetry and Remote Sensing 60(2), pp. 81-90.

Reulke, R., Tempelmann, U., Stallmann, D., Cramer, M. and Haala, N., 2004. Improvement of spatial resolution with staggered arrays as used in the airborne optical sensor ADS40. In: Proceedings of the XXth ISPRS Congress.

USAF, 1959. Military Standard Photographic Lenses. MIL-STD150A.

Valenzuela, Á. Q. and Reyes, J. C. G., 2019. Basic spatial resolution metrics for satellite imagers. IEEE Sensors Journal 19(13), pp. 4914-4922.

Wenzel, K., Rothermel, M., Fritsch, D. and Haala, N., 2013. Image acquisition and model selection for multi-view stereo. ISPRS - International Archives of the Photogrammetry, Remote Sensing and Spatial Information Sciences XL-5/W1, pp. 251-258.

Wg EMVA, E. ., 2016. Emva standard 1288 release 3.1.

Williams, C. S. and Becklund, O. A., 1989. Introduction to the optical transfer function. Wiley New York etc.

Wohlfeil, J., Strackenbrock, B. and Kossyk, I., 2013. Automated high resolution $3 \mathrm{~d}$ reconstruction of cultural heritage using multiscale sensor systems and semi-global matching. In: The International Archives of the Photogrammetry, Remote Sensing and Spatial Information Sciences, Cape Town, South Africa, Vol. XL4/W4, pp. 37-43. 